\title{
SUBCONTRACTING MANAGEMENT HANDBOOK
}

FIRST EDITION - 1988

\section{DEFENSE SYSTEMS MANAGEMENT COLLEGE FORT BELVOIR, VIRGINIA}

